\title{
Relationships Between Reactions of Sweet Corn Hybrids to Stewart's Wilt and Incidence of Systemic Infection by Erwinia stewartii
}

\author{
P. M. Michener, N. D. Freeman, and J. K. Pataky, Department of Crop Sciences, University of Illinois, Urbana \\ 61801
}

\begin{abstract}
Michener, P. M., Freeman, N. D., and Pataky, J. K. 2003. Relationships between reactions of sweet corn hybrids to Stewart's wilt and incidence of systemic infection by Erwinia stewartii. Plant Dis. 87:223-228.

Relationships between the reactions of sweet corn hybrids to Stewart's wilt and the incidence of natural, systemic infection by Erwinia stewartii differed among trials in which the prevalence of Stewart's wilt differed. Systemic Stewart's wilt infection was assessed for 262, 296, and 245 hybrids planted in seven trials in central Illinois in June and July 1998, 1999, and 2000, respectively. Incidence of systemic infection was calculated in each trial for all hybrids in each of nine categories of Stewart's wilt reactions (i.e., $1=$ resistant and $9=$ susceptible). When mean incidence was about $5 \%$, incidence ranged from about 1 to $8 \%$ on resistant to moderately susceptible hybrids, but incidence was nearly $30 \%$ on susceptible hybrids. When mean incidence ranged from 10 to $16 \%$, the relationships between hybrid reactions and incidence were explained by exponential or polynomial regressions. Incidence was less than $10 \%$ for hybrids with resistant and moderately resistant reactions, and incidence was greater than $15 \%$ for moderately susceptible to susceptible hybrids. When mean incidence was near $50 \%$, the relationship was linear. Incidence was about $18 \%$ for resistant hybrids and about $80 \%$ for susceptible hybrids. Incidence increased about $8 \%$ for each class of hybrid reaction from 1 to 9 . The influence of resistance on the development of systemic infection at very early seedling growth stages also was evaluated in six greenhouse trials. A highly resistant hybrid, Bonus, was systemically infected in two of six greenhouse trials when seedlings were inoculated prior to the V3 growth stage; however, systemic infection was not as severe as on a susceptible hybrid, Jubilee. Systemic infection was more severe on Bonus when plants were inoculated at earlier growth stages between VE and V3. The resistant hybrid Bonus was not systemically infected when inoculated after the V4 growth stage except for one greenhouse trial when all Stewart's wilt ratings were higher than usual. Hybrid reactions to Stewart's wilt affected the incidence of systemic infection in field situations and they affected the growth stage at which resistance effectively prevented systemic movement of $E$. stewartii within plants in greenhouse trials. This information can be used to determine more effectively when to apply other control measures, such as insecticidal seed treatments.
\end{abstract}

Stewart's bacterial wilt, caused by $E r$ winia stewartii (Smith) Dye (syn. Pantoea stewartii), is an economically important disease of sweet corn. Corn flea beetles, Chaetocnema pulicaria, are the vectors of E. stewartii (15). Stewart's wilt is endemic where flea beetles overwinter in the Mid-Atlantic States, the Ohio River Valley, and the southern portion of the United States Corn Belt,

Corresponding author: J. K. Pataky

E-mail: j-pataky@uiuc.edu

Partial funding provided by the Illinois Council on Food and Agricultural Research (CFAR) Grant No. 99I-016-3 and the Midwest Food Processors Association.

Accepted for publication 30 September 2002.

Publication no. D-2002-1219-01R

(C) 2003 The American Phytopathological Society including parts of Indiana, Illinois, Iowa, and Missouri (14).

Stewart's wilt can cause considerable economic damage to sweet corn hybrids with moderate to susceptible reactions $(1,5,12,17)$. Several sweet corn hybrids grown commonly for processing and many early-maturing sweet corn hybrids are susceptible (8). Yield losses due to Stewart's wilt are associated with systemic infection (5). Yield of susceptible or moderately susceptible hybrids is reduced substantially when seedlings are systemically infected as the result of inoculation or natural infection prior to approximately the seven-leaf stage $(1,5,12,17)$. Yield is not reduced as much when these hybrids are infected at the seven- to nine-leaf stages or thereafter (17). Yield of resistant or moderately resistant hybrids is affected less adversely than yield of susceptible hybrids regardless of the growth stage at which plants are infected $(5,12,17)$, primarily because resistant hybrids are systemically infected less frequently than susceptible hybrids.

The ability of E. stewartii to infect plants systemically is affected by host reactions and growth stage at the time of infection $(17,18)$. Susceptible hybrids can be infected systemically by $E$. stewartii as late as the five- to seven-leaf stages. Systemic infection of resistant hybrids is rare after early seedling growth stages because resistance restricts the movement of $E$. stewartii in the xylem (2); however, the growth stage at which resistance begins to restrict the movement of E. stewartii and effectively control Stewart's wilt is not known precisely. Systemic infection at early growth stages (e.g., prior to the threeleaf stage) can result in death of the main stalk, presumably because the growing point is infected (10). Ears are not produced when main stalks of plants are killed. When flea beetles were abundant in 1995, systemic infection and main stalk death were frequent even on sweet corn hybrids with moderate resistance to Stewart's wilt (10).

Incidence of systemic infection can be reduced by controlling the vector, $C$. pulicaria, or by growing resistant hybrids. Insecticidal seed treatments kill flea beetles (7) and decrease the incidence of systemic Stewart's wilt by 50 to $80 \%$ (13). The economic benefit of insecticidal seed treatments depends on the abundance of corn flea beetles during the early stages of crop growth, the level of hybrid resistance, the monetary value of the crop, and the cost of the seed treatment. Insecticidal seed treatments probably are economical on sweet corn grown for fresh market in situations where the incidence of systemically infected plants exceeds 3 to $5 \%$ in the absence of seed treatments (9). Insecticidal seed treatments probably are economical on sweet corn grown for processing in situations where the incidence of systemically infected plants exceeds 5 to $10 \%$ in the absence of seed treatments (9).

Although it is widely recognized that Stewart's wilt-resistant hybrids are infected systemically less often than susceptible hybrids, the relationships between hybrid reactions to Stewart's wilt and incidence of systemic infection are not well documented. Stewart's wilt reactions 
have been assessed for more than 2,000 sweet corn hybrids based on severity of symptoms following inoculation in disease nurseries at the University of Illinois (8). Reactions of hybrids range from resistant to highly susceptible. Further information about the relationships between reactions of hybrids to Stewart's wilt and incidence of natural, systemic infection could be useful in selecting control strategies for Stewart's wilt, such as identifying hybrids for which insecticidal seed treatments are an economical method of decreasing disease incidence.

The objectives of this research were to evaluate relationships between sweet corn hybrid reactions to Stewart's wilt and incidence of natural, systemic infection by $E$. stewartii and to evaluate if host resistance influences the development of systemic Stewart's wilt at very early stages of plant growth.

\section{MATERIALS AND METHODS}

Reactions of hybrids to Stewart's wilt. Reactions of hybrids to Stewart's wilt were determined previously in the University of Illinois sweet corn hybrid disease nurseries (8). In these nurseries, plants were inoculated with E. stewartii and severity of symptoms was rated from 1 to 9. Hybrid reactions to Stewart's wilt were classified as resistant (R), moderately resistant (MR), moderate (M), moderately susceptible (MS), and susceptible (S) based on mean ratings of Stewart's wilt severity in each individual nursery and various statistical measures of those data, including, standard deviations from trial means ( $z$ scores), separations based on multiple comparison tests (BLSD), and multivariate clustering procedures (12). The overall reaction of a hybrid to Stewart's wilt then was determined as an average of its reaction in each of the nurseries in which it was evaluated (i.e., various hybrids were evaluated in 3 to 17 individual disease nurseries from 1984 to $2000 ; 8)$. In order to calculate the average reaction of a hybrid to Stewart's wilt, disease reactions from individual nurseries were assigned numerical values from 1 to 9 , where: $1=$ resistant, $3=$ moderately resistant, $5=$ moderate, $7=$ moderately susceptible, and $9=$ susceptible. Thus, the 1-to-9 values assigned to hybrid reactions (ranging from $R$ to $S$ ) were not the same as the 1-to-9 rating scale used to assess the severity of Stewart's wilt symptoms in any particular disease nursery.

Incidence of systemic infection and severity of Stewart's wilt. Incidence of natural, systemic Stewart's wilt infection was assessed in seven trials planted after 1 June (late-planted) at the University of Illinois South Farms, Urbana. Trials were planted 30 June 1998; 22 June and 6 July 1999; and 6, 18, and 29 June and 6 July 2000. Trials included 262, 296, and 245 sweet corn hybrids in 1998, 1999, and 2000, respectively. The experimental de- sign for each trial was a split plot of a randomized complete block (RCB) with three replicates. Each trial was split into two main blocks of supersweet hybrids and normal or sugary enhanced hybrids. Each experimental unit was a single 4-m row with approximately 16 plants.

The number of plants and the number of systemically infected plants per row were counted once between the three- and seven-leaf stages in six trials and twice at the two- and seven-leaf stages in the trial planted 18 June 2000. Plants were recorded as systemically infected by E. stewartii if long, linear, chlorotic streaks were observed on multiple leaves or if the main stalk was dead and Stewart's wilt symptoms were evident on tillers. The incidence of systemic Stewart's wilt infection was calculated as a percentage for each hybrid in each trial.

The incidence of systemic Stewart's wilt infection was calculated for each reaction category (i.e., 1 to 9 corresponding to $\mathrm{R}$ to S) in each trial as a weighted mean of all hybrids in that reaction category. Means and standard errors were weighted by the number of plants of a hybrid in a reaction category per replicate. Relationships between the incidence of systemic infection and hybrid reactions were assessed by regression where $Y=$ incidence of systemically infected plants and $X=$ hybrid reaction to Stewart's wilt from 1 to 9. Regression analyses were done using GLM or REG procedures of SAS (Statistical Analysis Systems, Cary, NC).

Levels of Stewart's wilt in each trial were categorized arbitrarily based on the mean incidence of systemically infected plants. Trials with incidence less than 10 , from 10 to 20, from 20 to 40, and greater than $40 \%$ were considered to have low, moderate, moderately high, and high levels of Stewart's wilt, respectively.

Using the eight sets of incidence data from these seven trials, the probability of observing 0 to 5,5 to 10,10 to 15 , or more than $15 \%$ incidence of systemically infected plants was calculated from relative frequencies for hybrids with reactions of R/MR (1 to 2 ), MR/M (3 to 4), M/MS (5 to 6), MS/S (7 to 8), and S (9). These ranges of systemic infection were selected as being representative of situations where the probable economic value of seed treatment insecticides was very little, slight, moderate, and substantial.

Plant age and systemic infection. Six greenhouse trials were performed to evaluate the influence of resistance on systemic infection at very early stages of plant growth. The treatment design was a factorial of two sweet corn hybrids (Bonus and Jubilee) and seven to nine ages of plants ranging from 7 to 32 days after planting. Bonus is one of the sweet corn hybrids most resistant to Stewart's wilt. Jubilee is one of the most susceptible. Seed of each hybrid were planted at 2- to 3-day intervals for 15 to 28 days. The experimental design for each trial was a split plot of an RCB with three replicates. In the first three trials, an experimental unit consisted of about 60 plants growing in a 30-by-60-by-7-cm flat. In the last three trials, each experimental unit consisted of about 20 plants in each of two plastic pots, $20 \mathrm{~cm}$ in diameter by $25 \mathrm{~cm}$ deep. Plant ages were manipulated by planting dates which were main plots. Bonus or Jubilee were planted in subplots.

Inoculum was produced from a m2ixture of isolates of E. stewartii collected from infected corn leaves in Illinois in 1998 and 1999. Isolates were maintained as a bulked population on infected corn grown in the greenhouse. Inoculum was prepared by transferring 5 to 10 surface-sterilized, 1$\mathrm{cm}^{2}$ pieces of infected leaf tissue to 1 liter of nutrient broth in a 2-liter Erlenmeyer flask. Cultures were incubated at room temperature on shakers for approximately $15 \mathrm{~h}$ before being used as inoculum.

Seedlings were inoculated twice in each trial, 7 to 10 days after the last planting and 1 or 2 days later. When plants were inoculated, growth stage was recorded by counting the total number of visible leaves (16). Each seedling was wounded by inserting a $0.85-\mathrm{mm}$ (i.e., 22-gauge) hypodermic needle into the base of a plant near the tissues on which flea beetles usually feed. Plants were wounded in this manner three to five times and approximately 0.5 $\mathrm{ml}$ of inoculum was injected into the wounds.

Severity of symptoms was rated 2 to 3 weeks after inoculation. Each plant was rated on a 1-to-9 scale where $1=$ no spread or symptoms; 2 = little spread, with chlorosis, and necrosis within $2 \mathrm{~cm}$ of inoculation wounds; 3 = limited spread, with water-soaking, chlorosis, and necrosis within 2 to $6 \mathrm{~cm}$ of inoculation wounds; 4 $=$ abundant spread, with water-soaking chlorosis, and necrosis throughout inoculated leaves; 5 = limited systemic infection of noninoculated leaves; $6=$ moderate water-soaking chlorosis, necrosis, and wilting of 25 to $50 \%$ of the total leaf area, including noninoculated leaves; $7=$ severe chlorosis, necrosis, and wilting of 50 to $70 \%$ of the total leaf area; $8=$ severe chlorosis and necrosis of 75 to $90 \%$ of the total leaf area; and $9=$ dead or nearly dead plants. Means for each experimental unit were calculated from individual plant ratings.

The effects of hybrids and plant ages were assessed by analysis of variance (ANOVA) with a factorial treatment design. Stewart's wilt ratings were regressed on plant age (days after planting) for each hybrid in each trial.

\section{RESULTS}

Incidence of systemic infection and severity of Stewart's wilt. Mean incidence of systemic infection ranged from about 5 
to $50 \%$ in the seven trials (Table 1 ). In the trial planted 18 June 2000, incidence increased from about 16 to $25 \%$ between ratings at the two- and seven-leaf stages. Incidence was below $10 \%$ in the trial planted 6 June 2000; between 10 and 20\% in four trials (6 July 2000, 6 July 1999, 29 June 2000, and 18 June 2000; two-leaf stage); between 20 and $40 \%$ in two trials (30 June 1998 and 18 June 2000; sevenleaf stage); and above $40 \%$ in the trial planted 22 June 1999. Relationships between incidence of systemic infection and hybrid reaction to Stewart's wilt varied among trials with different levels of Stewart's wilt (Figs. 1 to 3 ).

In the 6 June 2000 trial, mean incidence of Stewart's wilt was low (i.e., about $5 \%$ ). The relationship between incidence of systemic infection and hybrid reaction to Stewart's wilt was linear except for susceptible hybrids (Fig. 1). Incidence was about $30 \%$ for susceptible hybrids. Incidence increased from about $1 \%$ for resistant hybrids to about $8 \%$ for moderately susceptible hybrids.

In four trials with a moderate level of Stewart's wilt (i.e., mean incidence from 10 to $16 \%$ ), relationships between incidence of systemic infection and hybrid reaction to Stewart's wilt were described by exponential or polynomial regressions (Fig. 2). Incidence was less than $10 \%$ for hybrids with resistant to MR/M reactions, except for the two-leaf assessment of the 18 June 2000 trial. In all trials with a moderate level of Stewart's wilt, incidence was greater than $15 \%$ for hybrids with MS to S reactions. Incidence also was greater than $15 \%$ for hybrids with $\mathrm{M} / \mathrm{MS}$ reactions at the two-leaf assessment in the 18 June 2000 trial.

In two trials with a moderately severe amount of Stewart's wilt (i.e., mean incidence near $25 \%$ ), relationships between incidence of systemic infection and hybrid reaction were described by polynomial regressions (Fig. 2). Incidence was less than $10 \%$ for hybrids with $\mathrm{R}$ and $\mathrm{R} / \mathrm{MR}$ reactions and for MR hybrids in the 30 June 1998 trial. Incidence was about 20\% or more for hybrids with $\mathrm{M}$ to $\mathrm{S}$ reactions.

Stewart's wilt was severe in the 22 June 1999 trial. Mean incidence was about 50\%. The relationship between incidence of systemic infection and hybrid reaction was linear (Fig 3). Incidence was greater than $15 \%$ for all hybrids. Incidence increased about $8 \%$ for each class of hybrid reaction.

Incidence of systemic infection of hybrids with $\mathrm{R}$ and $\mathrm{R} / \mathrm{MR}$ reactions (rating 1 to 2) was less than $10 \%$ in seven and six trials, respectively (Table 2). Incidence was less than $10 \%$ for hybrids with MR and $\mathrm{MR} / \mathrm{M}$ reactions (rating 3 to 4 ) in five and four trials, respectively. Thus, the probability of having less than $10 \%$ incidence of natural, systemic infection in these late-planted trials from 1998 to 2000 was 0.81 and 0.55 for hybrids with $\mathrm{R}$ (rating 1 to 2) and MR (rating 3 to 4) reactions to Stewart's wilt, respectively. For hybrids with $\mathrm{M}$ and $\mathrm{M} / \mathrm{MS}$ reactions (rating 5 to 6 ), incidence was greater than $10 \%$ in five and seven trials, respectively. Incidence was greater than $15 \%$ for hybrids with MS to $\mathrm{S}$ reactions in seven or eight trials (Table 2). The probability of more than $10 \%$ incidence was $0.75,0.88$, and 1.0 for hybrids with M (5 to 6 ), MS (7 to 8), and S (rating 9) reactions to Stewart's wilt, respectively.

Plant age and systemic infection. In all trials, the main effect of hybrids was highly significant $(P<0.0001)$ and the hybrid-plant age interaction term was not significant. Stewart's wilt ratings were higher for Jubilee than for Bonus in each trial (Fig. 4). The main effect of plant age

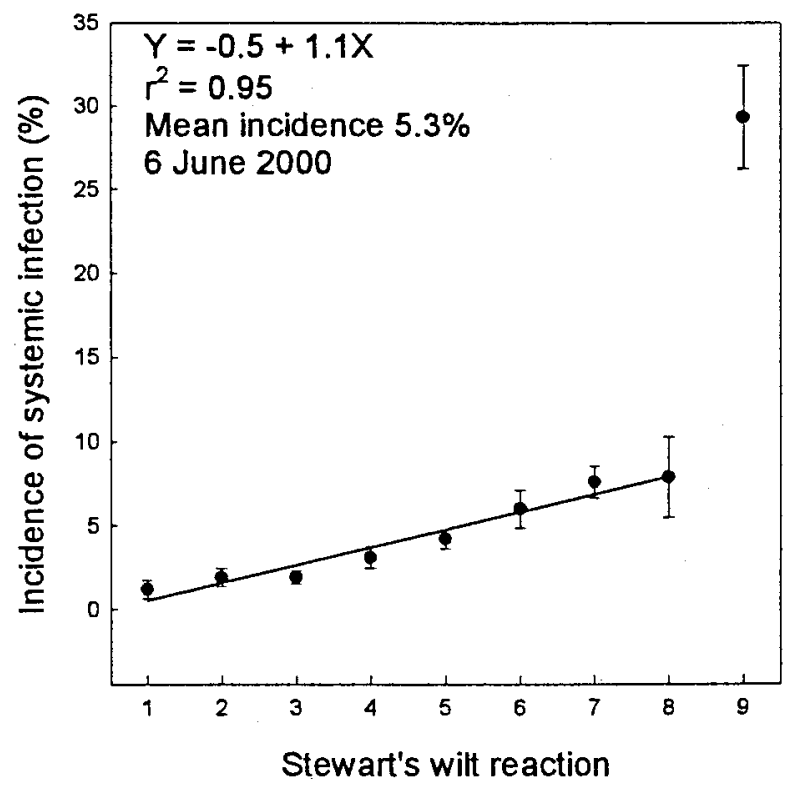

Fig. 1. Relationship between Stewart's wilt reactions of sweet corn hybrids and incidence of natural, systemic infection in a trial with a mean incidence near 5\%, planted 6 June 2000. Stewart's wilt reactions of hybrids: 1 = resistant, $3=$ moderately resistant, $5=$ moderate, 7 = moderately susceptible, and $9=$ susceptible. Weighted standard errors based on number of plants per replicate per reaction category.

Table 1. Mean incidence (\%) of natural, systemic Stewart's wilt infection of sweet corn hybrids with various reactions to Erwinia stewartii in trials planted in June or early July in Urbana, IL in 1998, 1999, and 2000a

\begin{tabular}{|c|c|c|c|c|c|c|c|c|c|c|c|}
\hline \multirow[b]{2}{*}{ Reaction $^{b}$} & \multicolumn{2}{|r|}{1998} & \multicolumn{3}{|c|}{1999} & \multicolumn{6}{|c|}{2000} \\
\hline & $n$ & 30 June (\%) & $n$ & 22 June (\%) & 6 July (\%) & $n$ & 6 June (\%) & 18 June $(\%)^{c}$ & 18 June $(\%)^{d}$ & 29 June (\%) & 6 July (\%) \\
\hline $1(\mathrm{R})$ & 8 & 8.5 & 8 & 17.4 & 0.4 & 8 & 1.2 & 3.8 & 6.3 & 3.8 & 5.1 \\
\hline 2 (R/MR) & 16 & 6.3 & 27 & 22.6 & 1.6 & 30 & 1.9 & 4.7 & 11.9 & 6.4 & 4.5 \\
\hline 3 (MR) & 22 & 11.4 & 35 & 35.0 & 3.3 & 40 & 1.9 & 8.0 & 13.0 & 6.4 & 5.6 \\
\hline $4(\mathrm{MR} / \mathrm{M})$ & 22 & 12.0 & 45 & 42.8 & 4.1 & 43 & 3.1 & 13.0 & 20.1 & 9.3 & 7.1 \\
\hline $5(\mathrm{M})$ & 70 & 19.7 & 46 & 53.0 & 7.2 & 51 & 4.2 & 15.9 & 42.8 & 11.9 & 8.9 \\
\hline 6 (M/MS) & 57 & 25.9 & 53 & 59.4 & 11.7 & 27 & 6.0 & 16.3 & 29.6 & 15.7 & 13.7 \\
\hline 7 (MS) & 34 & 32.4 & 34 & 67.2 & 19.0 & 24 & 7.6 & 27.8 & 43.1 & 21.3 & 16.3 \\
\hline $8(\mathrm{MS} / \mathrm{S})$ & 19 & 46.7 & 28 & 71.6 & 29.6 & 8 & 7.9 & 30.3 & 50.1 & 22.5 & 21.3 \\
\hline $9(\mathrm{~S})$ & 14 & 63.0 & 20 & 79.5 & 48.6 & 14 & 29.3 & 46.7 & 66.0 & 36.1 & 32.1 \\
\hline Mean & $\ldots$ & 23.9 & $\ldots$ & 50.9 & 11.8 & $\ldots$ & 5.3 & 15.6 & 25.1 & 12.6 & 10.3 \\
\hline
\end{tabular}

${ }^{a}$ Number of hybrids $(n)$ in each category for trials in 1998, 1999, and 2000 with about 45 plants per hybrid per trial.

${ }^{\mathrm{b}}$ Hybrid reactions from resistant (1) to susceptible (9) based on evaluations in disease nurseries from 1984 to 2000 (8). R = resistant, MR = moderately resistant, $\mathrm{M}=$ moderate, $\mathrm{MS}=$ moderately susceptible, and $\mathrm{S}=$ susceptible.

${ }^{c}$ Incidence evaluated at two-leaf stage in trial planted 18 June 2000.

${ }^{d}$ Incidence evaluated at seven-leaf stage in trial planted 18 June 2000. 
was highly significant in trials 4,5 , and 6 , when plants were grown in 20-cm-diameter pots. Plant age was significant with a higher probability $(0.01<P<0.08)$ of incorrectly rejecting the null hypothesis in trials 1,2 , and 3 , when plants were grown in flats that were $7 \mathrm{~cm}$ deep. In each trial, Stewart's wilt ratings were higher when plants were inoculated at an earlier growth stage (i.e., younger plant age).

In trials 4 to 6 , Stewart's wilt ratings for Jubilee ranged from about 3.5 to 8 (Fig. 4), which is typical of field trials for this susceptible hybrid. Jubilee usually is infected systemically by $E$. stewartii (i.e., ratings of 4 and above). Ratings in trials 4 to 6 usually were above 5 for Jubilee inoculated at the V1 to V4 growth stages (i.e., less than 18 days after planting). Ratings usually were between 3.5 and 6 for Jubilee inoculated at the V4 to V6 growth stages (i.e., 18 to 32 days after planting).

In trials 5 and 6, Stewart's wilt ratings for Bonus were above 3.5 for plants inoculated at the V1 to V3 growth stages (i.e., 8 to 14 days after planting). When Bonus was inoculated after the V4 growth stage (i.e., about 20 days after planting), ratings were below 3 (nonsystemic infection) in all

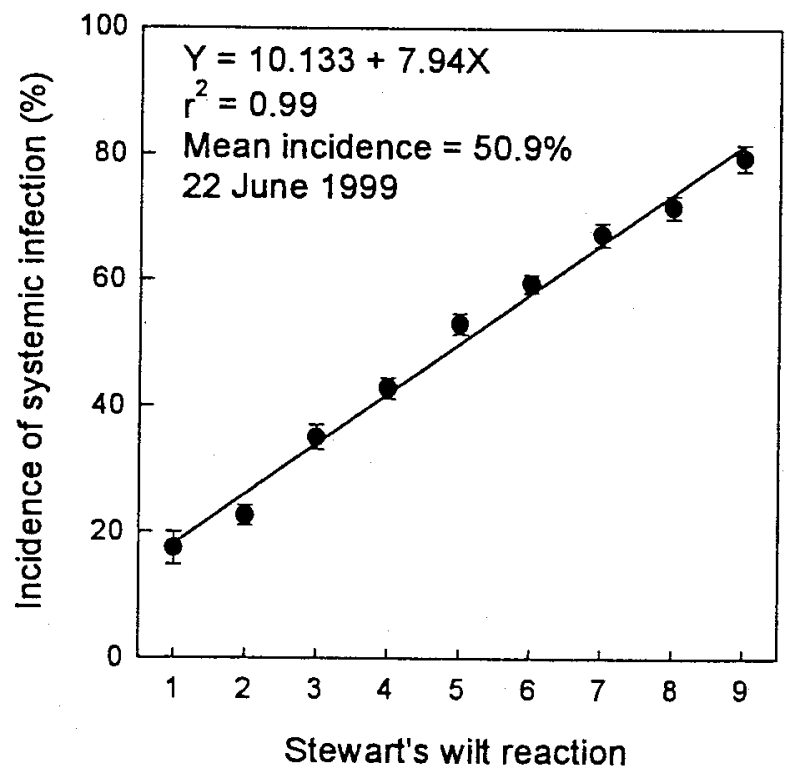

Fig. 3. Relationship between Stewart's wilt reactions of sweet corn hybrids and incidence of natural, systemic infection in a trial with a mean incidence near 50\%, planted 22 June 1999. Stewart's wilt reactions of hybrids: $1=$ resistant, $3=$ moderately resistant, $5=$ moderate, $7=$ moderately susceptible, and $9=$ susceptible. Weighted standard error based on number of plants per replicate per reaction category.

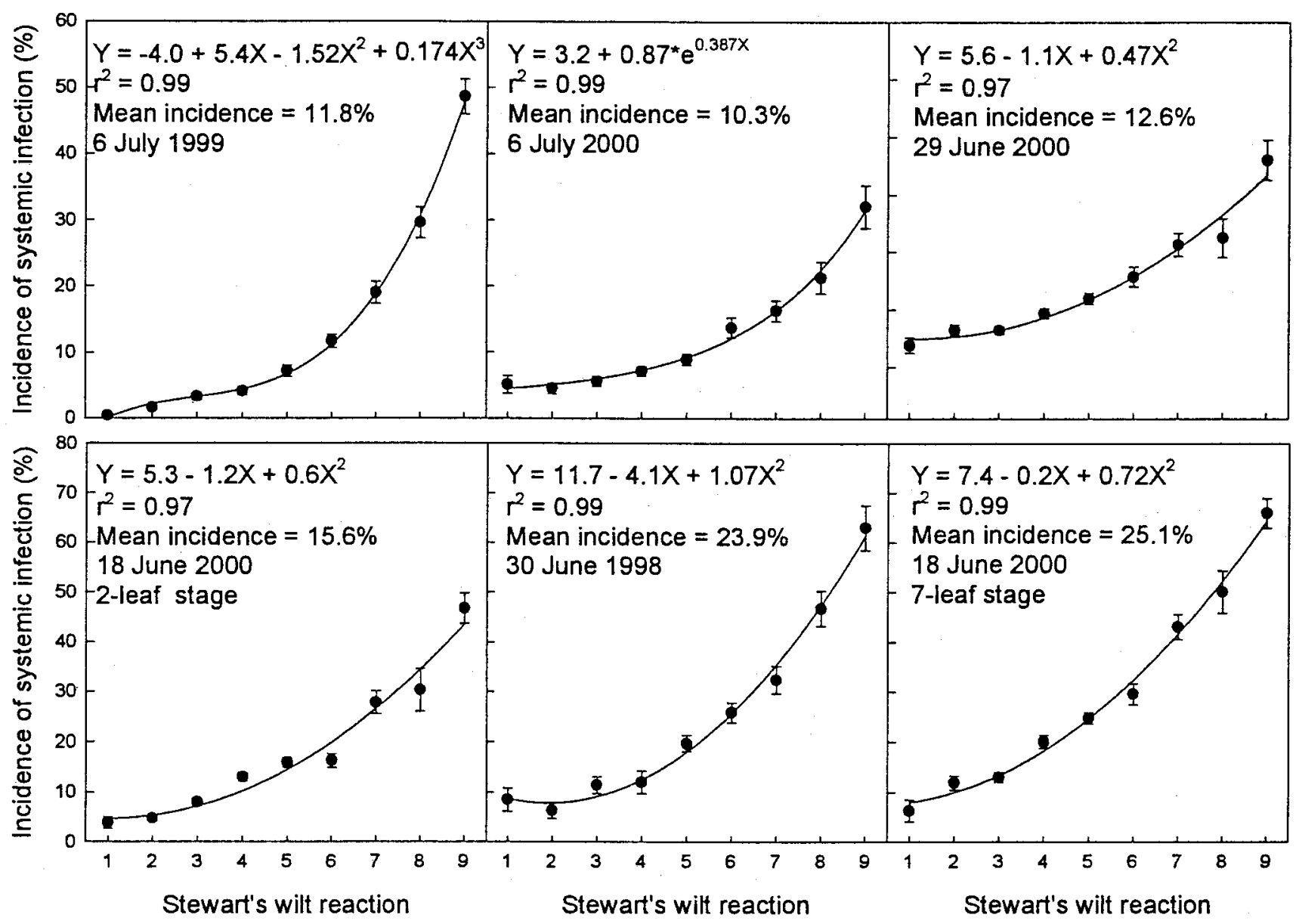

Fig. 2. Relationships between Stewart's wilt reactions of sweet corn hybrids and incidence of natural, systemic infection in trials with mean incidence ranging from 10 to $25 \%$, planted in June or July, 1998 to 2000. Stewart's wilt reactions of hybrids: $1=$ resistant, $3=$ moderately resistant, $5=$ moderate, $7=$ moderately susceptible, and $9=$ susceptible. Weighted standard errors based on number of plants per replicate per reaction category. 
trials except trial 4, when Bonus was inoculated after the V4 growth stage (i.e., about 20 days after planting).

In trials 1 and 2, Stewart's wilt ratings for Jubilee were substantially lower than typical ratings recorded for this hybrid in field trials. In trial 3, ratings for Jubilee were within the normally observed range, but ratings for Bonus were higher than usual. Possibly, Stewart's wilt development in trials 1 to 3 was affected by plant growth. Roots were restricted in the first three trials because plants were grown in flats that were only $7 \mathrm{~cm}$ deep.

\section{DISCUSSION}

The relationship between hybrid reactions to Stewart's wilt and incidence of systemic infection of seedlings was not the same among trials in which the prevalence of Stewart's wilt differed. Relationships were linear when mean incidence was low (about 5\%) or high (greater than 25\%) and curvilinear when mean incidence was moderate (from 10 to $25 \%$ ). Hybrids with $\mathrm{R}$ or $\mathrm{MR}$ reactions provided adequate control (less than approximately $10 \%$ incidence) except when Stewart's wilt mean incidence was high (about 50\%); whereas, hybrids with M to MS reactions had more than $10 \%$ systemically infected plants unless levels of Stewart's wilt were very low (mean incidence about 5\%). Incidence of sys- temic infection was high for susceptible hybrids even when mean incidence of Stewart's wilt was very low.

Braun (2) observed that resistance restricts the systemic movement of $E$. stewartii within the host. The growth stage of the host and the location of flea beetles feeding sites may affect the efficacy of resistance. When populations of flea beetles are large, plants may be infected at very early growth stages. In two of our greenhouse trials, a highly resistant hybrid, Bonus, was infected systemically when seedlings were inoculated prior to the V3 growth stage although severity of systemic infection decreased as plants were inoculated from the V1 to V3 stages. Also, systemic infection was not as severe on Bonus as on the susceptible hybrid, Jubilee. The results of these greenhouse trials led us to believe that incidence of natural, systemic Stewart's wilt on resistant to moderately resistant hybrids in our field trials was the result of flea beetle feeding during the first 2 weeks after planting (i.e., prior to the V2 growth stage). During this period, flea beetles fed on plant tissues that were in close proximity to the growing point. When these feeding sites were infected, $E$. stewartii needed to move only a few centimeters to cause systemic infection or main stalk death. At later growth stages, infection sites were further from the growing point and resistance was effective, proba-

Table 2. Number of trials of eight total in which incidence of natural, systemic Stewart's wilt was less than 5,5 to 10,10 to 15 , or greater than $15 \%$ for hybrid with various reactions to Stewart's wilt

\begin{tabular}{lcccc}
\hline & \multicolumn{4}{c}{ No. of trials with incidence of systemic infection in ranges of: } \\
\cline { 2 - 5 } Reaction $^{\mathbf{a}}$ & $<\mathbf{5 \%}$ & $\mathbf{5 - 1 0 \%}$ & $\mathbf{1 0 - 1 5 \%}$ & $>\mathbf{1 5 \%}$ \\
\hline 1 (R) & 4 & 3 & 0 & 1 \\
2 (R/MR) & 4 & 2 & 1 & 1 \\
$3(\mathrm{MR})$ & 2 & 3 & 3 & 1 \\
$4(\mathrm{MR} / \mathrm{M})$ & 2 & 2 & 2 & 2 \\
5 (M) & 1 & 2 & 1 & 4 \\
(M/MS) & 0 & 1 & 2 & 5 \\
7 (MS) & 0 & 1 & 0 & 7 \\
8 (MS/S) & 0 & 1 & 0 & 7 \\
(S) & 0 & 0 & 0 & 8 \\
\hline
\end{tabular}

${ }^{\mathrm{a}} \mathrm{R}=$ resistant, $\mathrm{MR}=$ moderately resistant, $\mathrm{M}=$ moderate, $\mathrm{MS}=$ moderately susceptible, and $\mathrm{S}=$ susceptible.

bly because movement of E. stewartii was limited sufficiently.

The results from these studies can be used to better identify how levels of host resistance and insecticidal seed treatments can be integrated to reduce the incidence of systemic Stewart's wilt. Based on an estimated economic threshold of about $5 \%$ incidence of systemic infection and the relationships observed in our trials, seed of hybrids with $\mathrm{M}$ to $\mathrm{S}$ reactions should be treated with insecticides if Stewart's wilt is likely to be present. Seed of hybrids with R to MR reactions to Stewart's wilt should be treated only if flea beetle populations are expected to be large and a substantial number of plants are likely to be infected within the first 2 weeks after planting.

In the absence of accurate models to predict the occurrence of flea beetles, the probability of observing various levels of incidence of natural, systemic Stewart's wilt infection can be used to predict when insecticidal seed treatments or other control tactics might be economical on hybrids with known reactions to Stewart's wilt. Based on eight evaluations in seven trials of our field study, the probability of observing less than $5 \%$ incidence on hybrids with $\mathrm{R}, \mathrm{MR}$, and $\mathrm{M}$ reactions is 0.5 , 0.25 , and 0.13 , respectively. The probability of observing greater than $10 \%$ incidence on hybrids with MS or S reactions based on these trials is 0.81 and 1.0 respectively. Thus, we might expect insecticidal seed treatments to be economical on late-season plantings of MS or S hybrids a majority of the time when conditions are similar to central Illinois in 1998, 1999 and 2000, but economical on R hybrids only half the time.

Additional information about flea beetle population dynamics in relation to incidence of Stewart's wilt on sweet corn also could be used to integrate control tactics more effectively. Although populations of corn flea beetles were not monitored in our field study, our observations of a high incidence of Stewart's wilt in plantings from mid-June to early July corresponds

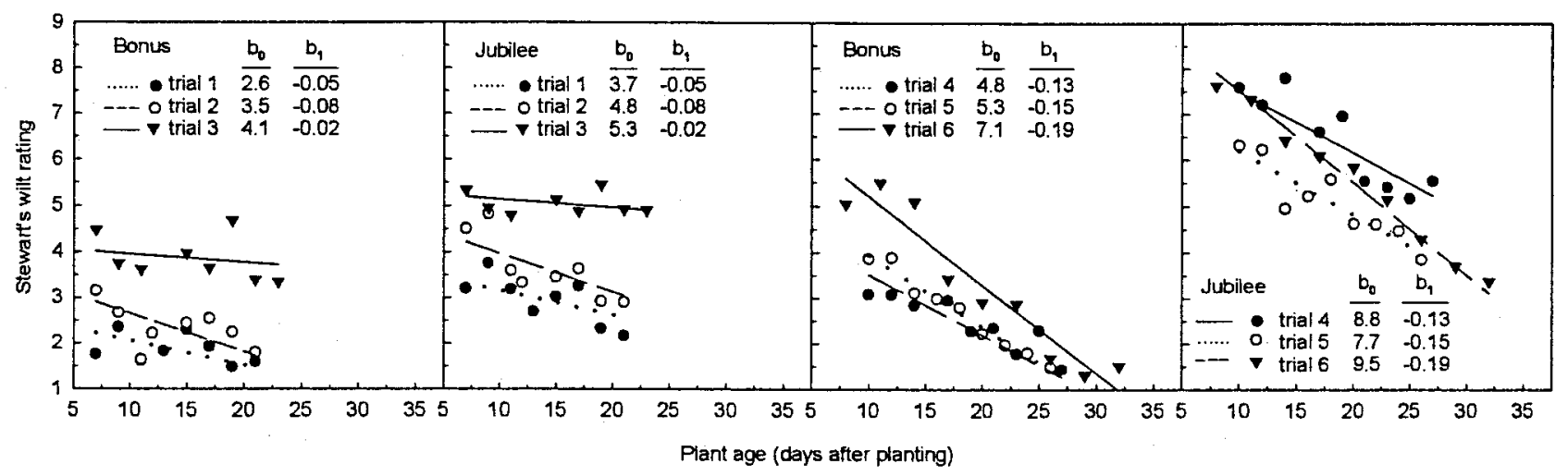

Fig. 4. Relationship between Stewart's wilt severity rating (1 to 9) and age of plants (days after planting) at inoculation for a susceptible sweet corn hybrid, Jubilee, and a resistant sweet corn hybrid, Bonus, planted in six greenhouse trials. 
with previous and recent surveys of flea beetle populations and Stewart's wilt incidence $(3,4,6,11)$. Recently, Esker et al. (4) observed three generations of corn flea beetles in Iowa. The first generation, comprising overwintering flea beetles, was detected in early to mid-May. The second and third generations were detected in early to mid-July and mid- to late August, respectively. Heichel et al. (6) reported two distinct broods of flea beetles in Connecticut in 1975 based on observations of sequential plantings of the susceptible sweet corn hybrid Jubilee. Incidence decreased from about 70 to $12 \%$ in sequential plantings at approximately 10-day intervals throughout May and the first 2 weeks of June 1975. Incidence increased to about $60 \%$ in plantings after 16 June 1975. Pataky et al. (11) reported an increase in incidence of systemic Stewart's wilt in four sequential plantings of sweet corn in 3 of 4 years between 1989 and 1992 in Urbana, IL. Initial trials were planted early to midMay and successive planting were made approximately every 2 weeks through midto late June. Incidence was highest in 1989, 1991, and 1992 when trials were planted between 15 and 30 June (11). Considering these temporal patterns for flea beetle populations and Stewart's wilt and based on the hybrid reaction-incidence relationships we observed in our study, the incidence of systemic Stewart's wilt on R and MR hybrids grown in the Midwest might be expected to exceed $5 \%$ in very early plantings (prior to 10 May) if the overwintering population of flea beetles is large and in very late plantings (after 10 June), but not in mid-season plantings (10 May to 10 June).

\section{LITERATURE CITED}

1. Ayers, J. E., MacNab, A. A., Tetrault, R. C., and Yocum, J. O. 1979. The influence of selected insecticides on yield and the incidence of Stewart's wilt in sweet corn. Plant Dis. Rep. 63:634-638.

2. Braun, E. J. 1982. Ultrastructure investigation of resistant and susceptible maize infected with Erwinia stewartii. Phytopathology 72:159-166.

3. Elliott, C., and Poos, F. W. 1940. Seasonal development, insect vectors, and host range of bacterial wilt of sweet corn. J. Agric. Res. 60:645-686.

4. Esker, P. D., and Nutter, F. W., Jr. 2002. Development and use of degree-day models to improve management of corn flea beetles in the Stewart's disease pathosystem on corn. (Abstr.) Phytopathology 92:S24.

5. Freeman, N. D., and Pataky, J. K. 2001. Levels of Stewart's wilt resistance necessary to prevent reductions in yield of sweet corn hybrids. Plant Dis. 85:1278-1284.

6. Heichel, G. H., Sands, D. C., and Kring, J. B. 1977. Seasonal patterns and reduction by carbofuran of Stewart's bacterial wilt of sweet corn. Plant Dis. Rep. 61:149-153.

7. Munkvold, G. P., McGee, D. C., and Iles, A. 1996. Effects of imidacloprid seed treatment of corn on foliar and Erwinia stewartii transmission by the corn flea beetle. Plant Dis. 80:747-749.

8. Pataky, J. K. 2000. Reactions of sweet corn hybrids to prevalent diseases. Pages 177192 in: Midwest Vegetable Variety Trial Report for 2000. Purdue Univ. AES
Bull. No. 798.

9. Pataky, J. K. 2002. Managing Stewart's wilt and common rust in sweet corn. Pages 85-87 in: Proc. 2002 Ohio Fruit Veg. Grow. Congr. Toledo, $\mathrm{OH}$.

10. Pataky, J. K., du Toit, L. J., Kunkel, T. E., and Schmitt, R. A. 1996. Severe Stewart's wilt in central Illinois on sweet corn hybrids moderately resistant to Erwinia stewartii. Plant Dis. 80:104.

11. Pataky, J. K., Hawk, J. A., Weldekidan, T., and Fallah Moghaddam, P. 1995. Incidence and severity of Stewart's bacterial wilt on sequential plantings of resistant and susceptible sweet corn hybrids. Plant Dis. 79:1202-1207.

12. Pataky, J. K., Headrick, J. M., and Suparyono. 1988. Classification of sweet corn hybrid reactions to common rust, northern leaf blight, Stewart's wilt, and Goss's wilt and associated yield reductions. Phytopathology 78:172-178.

13. Pataky, J. K., Michener, P. M., Freeman, N. D., and Weinzerl, R. A. 2000. Control of Stewart's wilt with seed treatment insecticides. Plant Dis. 84:1104-1108.

14. Pepper, E. H. 1967. Stewart's bacterial wilt of corn. Am. Phytopathol. Soc. Mono. No. 4.

15. Poos, F. W., and Elliott, C. 1936. Certain insect vectors of Aplanobacter stewartii. J. Agric. Res. 52:585-608.

16. Ritchie, S. W., Hanway, J. J., and Benson, G. O. 1986. How a corn plant develops. Iowa Coop. Ext. Serv. Spec. Rep. 48

17. Suparyono, and Pataky, J. K. 1989. Influence of host resistance and growth stage at time of inoculation on Stewart's wilt and Goss's wilt development and sweet corn hybrid yield. Plant Dis. 73:339-345.

18. Suparyono, and Pataky, J. K. 1989. Relationships between incidence and severity of Stewart's and Goss's bacterial wilts and yield of sweet corn hybrids. Crop Prot. 8:363-368. 